\title{
Machine Vision System for Evaluating Performance Measure of Laser Beam on Leather
}

\author{
Karen Vanessa Pennefather, Rajesh Masilamani A, S. Vasanth
}

\begin{abstract}
The manual inspection of leather is sluggish and labor-intensive tasks, they can become precarious in the entire production process. In the present study an attempt has been made to investigate the Heat Affected Zone (HAZ) of leather during the laser cutting process due to the thermal effect caused by a laser at the cut contour edges. To inspect this, a machine vision system is used to capture images of the laser cut edges and then process it using MATLAB to acquire the required information for assessing the quality of leather. The leather can be categorized as good, average or poor quality based on the acquired information for the respective application. As a result, the degree of burn of the leather was determined.
\end{abstract}

Index Terms: HAZ (Heat Affected Zone), Laser, Leather, Machine Vision, MATLAB, Quality.

\section{INTRODUCTION}

Leather being highly flexible in nature has been utilized several thousands of years for clothing and making accessories. Animal hide obtained consists of natural striations, scars, defects etc. The animal hide undergoes a number of processes called tanning before it can be made into a final finished product [2]. During tanning process, the properties of leather are altered such as protein structure, texture, and colour to some extent. With leather industry developing, a far more enhanced system is required to meet the demands of the present world. Leather being a non-metallic material, due to which only non-conductive cutting methodologies can be used to cut it. Availability of Laser technology has paved way to design a system which produces highly accurate, adaptive and efficient method to cut leather of the required shapes and dimensions.

In any production unit an inspection system is required to assure good quality products. The inspection systems are required at different levels. The most basic inspection systems are necessary immediately after tanning process, after the finished product and sometimes in between a specific process to inspect and maintain leather quality. In this paper the inspection system right after the leather is cut using a $2.5 \mathrm{~W}$ violet laser. Machine vision system is used for image acquisition, reconstruction, enhancement, filtering, segmentation and analysis.

Revised Version Manuscript Received on Jun 20, 2019.

Karen Vanessa Pennefather, Department of Mechatronics, SRM Institute of Science and Technology, Kattankulathur, India.

Rajesh Masilamani A, Department of Mechatronics, SRM Institute of Science and Technology, Kattankulathur, India.

S. Vasanth, Department of Mechatronics, SRM Institute of Science and Technology, Kattankulathur, India.

\section{BACKGROUND}

One of the main advantages of laser cutting is production at standard quality. Laser cutting also maintains production in shorter time with higher quality. Due to ability to apply CAD/CAM and CIM system in design, it is possible to manufacture absolutely identical designs. The lasers based on their power, intensity, speed of cut and various other parameters is being used for a range application such as laser printers, LASIK surgery, barcode scanners etc. [8]. The major disadvantage of using laser cutting on leather is the emergence of Heat Affected Zone (HAZ). The absorbed heat energy on the surface of the leather induces thermal stresses which vaporizes the material to minimal extent causing HAZ. The camera in general is a recording device which can be used for extracting information by storing, modifying and processing the captured image. In the present world high speed computes are utilized which means images can be processed at a faster rate and with the appropriate software such as Matlab with which the processes are made simplified and faster. In Matlab Image Processing toolbox is used to capture and process the images. For a good machine vision system the system has to be trained sufficiently high number of times in order to maintain precision. This paper emphasizes on a more efficient method for cutting leather and its inspection which would improve quality, increase the production rate of cut leather and economic factors of a company.

\section{EXPERIMENTAL SETUP}

The entire system is a gantry setup which is robust and compact in nature. This setup was chosen for ease of movement of laser. The frame of the setup is made of aluminium and acrylic based materials. Aluminium has high tensile strength and light in nature and acrylic is used for holding the frame together to maintain the dimensional stability of the structure. Figure 1 shows the experimental setup. The laser is vertically mounted on the setup which offers two degrees of freedom that is $\mathrm{X}$ and $\mathrm{Y}$ direction movement. The movement of the laser module is controlled by means of the stepper motors. In this system a total of 3 stepper motors are used.

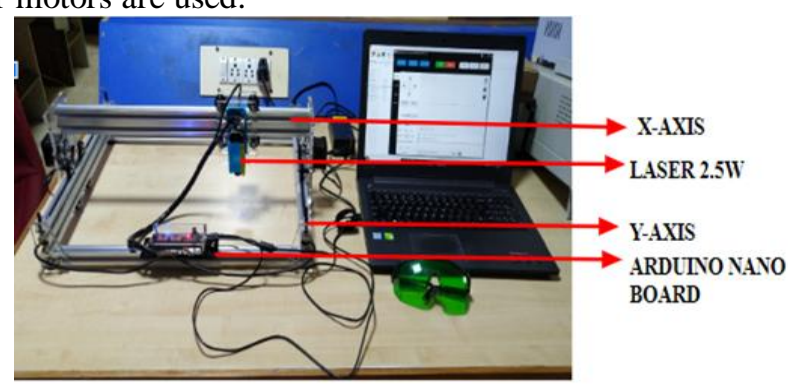

Figure 1 Experimental Setup 
Two steppers motors are used for the Y-axis movement of the laser and one motor is used for the $\mathrm{X}$-axis movement of the laser module. The dimensions- length, width, and height of the overall setup is $600 \mathrm{~mm}, 460 \mathrm{~mm}$ and $180 \mathrm{~mm}$ respectively. Table 1 shows the setup description and specifications.

\begin{tabular}{|c|c|c|}
\hline S.NO & DESCRIPTION & SPECIFICATIONS \\
\hline 1 & $\begin{array}{c}\text { Mechanical Setup Dimensions } \\
(\text { LxBxH) }\end{array}$ & $(600 \times 460 \times 180) \mathrm{mm}$ \\
\hline 2 & Working Range & $(380 \times 300) \mathrm{mm}$ \\
\hline 3 & Weight & $3.4 \mathrm{Kg}$ \\
\hline 4 & Control Board & Eleks Maker SE \\
\hline 5 & Power & $42 \mathrm{~V}, 5 \mathrm{~A}$ DC \\
\hline 6 & Maximum Travel Speed & $400 \mathrm{~mm} / \mathrm{s}$ \\
\hline 7 & Support Software & Elekscam, T2 laser \\
\hline
\end{tabular}

Table 1. Experimental Setup Description

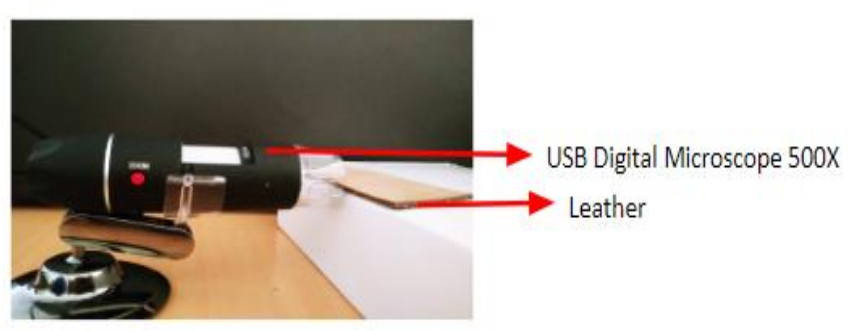

Figure 2 Firefly gt 800 handheld USB Microscope

A violet laser of $2.5 \mathrm{Watt}$ and $445 \mathrm{~nm}$ wavelength is used to cut the leather. The speed with which the laser and axis movement can be controlled by a programming Software. Figure 2 shows the digital USB Microscope camera which is used to capture the images of the contour burned edges of the leather. The background of the leather is ensured to be white because the presence of any other color would result in a dark shadow or dark effect on the images while capturing. Table 2 shows the camera description and specifications.

\begin{tabular}{|c|c|c|}
\hline S. No & DESCRIPTION & SPECIFICATION \\
\hline 1 & Light Source & 8 White Light LED \\
\hline 2 & Lens & Microscope Lens \\
\hline 3 & Magnification Ratio & $50 \mathrm{X}-500 \mathrm{X}$ \\
\hline 4 & Optimum Resolution & $640 \mathrm{x} 480$ \\
\hline 5 & Power Supply & USB Port(5V DC) \\
\hline 6 & Focus Range & 0mm to 40mm \\
\hline
\end{tabular}

Table 2. Camera Specification

The lighting is provided on top of the leather so that it facilitates accurate capturing of images. The camera is connected to a computer by means of the USB cable attached to the camera. The interfacing of the computer and camera takes place. The main features of this system is that there is no height limitation, the presence of 3 stepper motors facilitates faster movement of the laser, presence of heat sink in laser also reduces the thermal effect of the laser which in turn reduces the burning of the leather.

\section{MACHINE VISION SYSTEM}

The method of capturing, analyzing and segregating quality of leather based on images (or generally two- or three-dimensional modalities) reveals important contributions to this research. The appearance of leather changes significantly with imaging techniques, depending on the scale of analysis, imaging settings and physical characteristics. In this research we use Image Processing Toolbox for image acquisition, from which information is extracted and based on which decision is attained. This method only informs us about the physical characteristics from the image captured and doesn't speak about the chemical properties. This system was used to view, capture, and process the edges of manual and laser cut leather. The leather samples included buffalo. The darkening effects (HAZ- Heat Affected Zone) caused due to the thermal effect of the laser was viewed using a USB digital microscope $500 \mathrm{X}$. The images were captured in real time and compared to a set allowable range. The images (RGB) were converted to binary image using a set threshold value to determine the percentage of darkening in the whole image (laser cut edges). Image captured per frame was able to cover only $4 \mathrm{~mm}$ of the actual leather. The image was magnified and displayed approximately. 10 times the actual size.

A. Algorithm:

Step 1: Preview the list of cameras available.

Step 2: Set a variable with the desired camera.

Step 3: Capture and save the image with a variable

Step 4: Display the captured image.

Step 5: Eliminating the background region in the image.

Step 6: Conversion of image to binary image with a set threshold.

Step 7: Determine the size of the image matrix formed Step 8: Calculate the percentage of ones and zeros.

Step 9: Display the calculated percentage in real time.

Step 10: Using the if else case to categorizing the quality of leather based on the percentage of darkening as average, good, bad.

Formula Used to Determine the Level of Carbonization:

$\%$ of no Carbonization $=((\operatorname{sum}(\mathrm{BW}(\mathrm{BW}==1))) / \mathrm{A}) * 100$

$\%$ of Carbonization $=100-(\%$ of no Carbonization $)$

Where,

$\mathrm{BW}=$ binary image matrix.

sum $=$ sum of the number of ones in the matrix

$\mathrm{A}=$ the total matrix size of the image

\section{BLOCK DIAGRAM}

The figure 3 shows the block diagram of the laser setup and the camera setup. The stepwise procedure can be observed from the same, starting from the user block. 


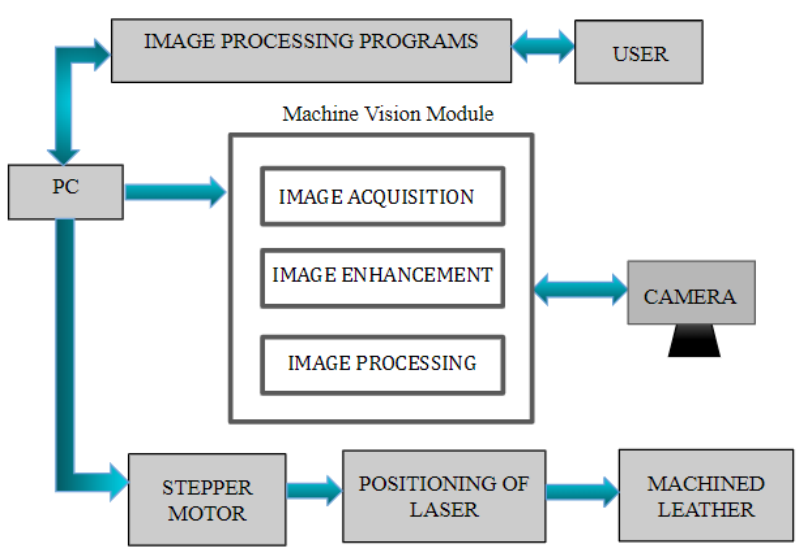

Figure 3. Block Diagram of the entire system

\section{CHEMICAL COMPOSITION OF LEATHER}

The chemical composition of the leather needs to be understood. The change in chemical properties of the leather changes the texture, color, overall quality and lifetime of the leather. Hence it is required that not much of the properties are changed while using the laser for the machining process. For this purpose a study was conducted on the chemical compounds present in the leather before and after the use of laser. The main aim of this study was to determine the level of carbonization effect of the laser on the leather. The experiment was conducted using EDX System. Table 3 shows the system specifications of the EDX system.

\begin{tabular}{|c|c|c|}
\hline S.N0 & DESCRIPTION & SPECIFICATIONS \\
\hline 1 & Maximum Magnification & $30,000 \mathrm{X}$ \\
\hline 2 & Resolution(SE) & $15 \mathrm{~nm}$ \\
\hline 3 & Resolution(BSE) & $20 \mathrm{~nm}$ \\
\hline 4 & Axis & 3-axis Stroke-X,Y,(Rotation) \\
\hline
\end{tabular}

Table 3. EDX System Specifications

The EDX System is an analytical technique used for the elemental analysis or chemical characterization of a sample. A high-energy beam of charged particles such as electrons or protons or a beam of X-rays, is focused into the sample being studied. At rest, the atoms of the sample are in ground state. Whereas, after incident of the beam they get excited and the electron that gets excited moves from its position and thus forming electron-hole pair. An electron from an outer, higher-energy shell then fills the hole, and the difference in energy between the higher-energy shell and the lower energy shell may be released in the form of an X-ray. The number and energy of the X-rays emitted from a specimen can be measured by an energy-dispersive spectrometer. As the energies of the X-rays are characteristic of the difference in energy between the two shells and of the atomic structure of the emitting element, EDS allows the elemental composition of the specimen to be measured. Figure 4. shows the chemical compounds in an manual cut buffalo leather.

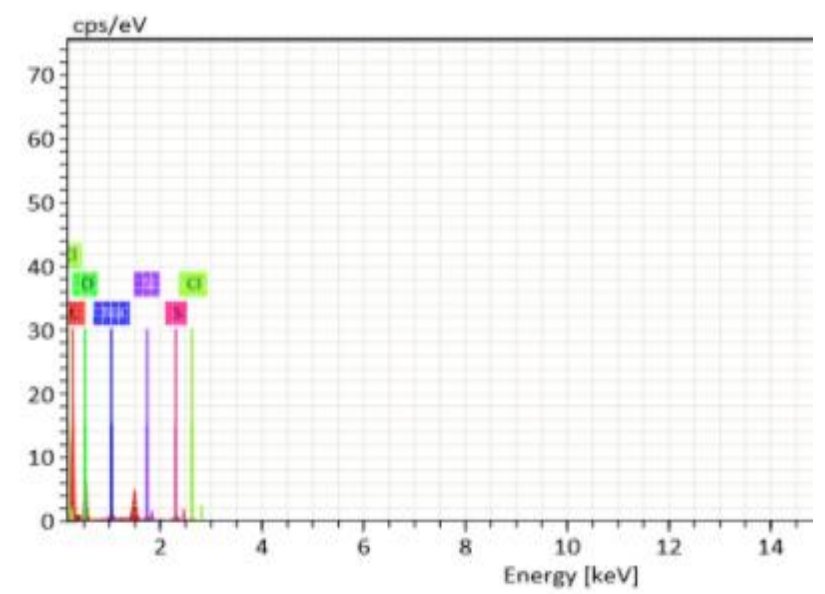

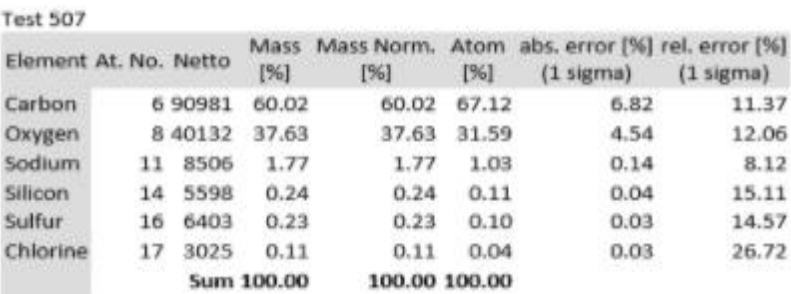

Figure 4. Chemical Compounds in a normal buffalo leather sample

Figure 5. Shows the chemical compounds present in the contour edge of the leather after the laser cut. The number of chemical compounds and their percentage can be seen in the table in figure 5 .

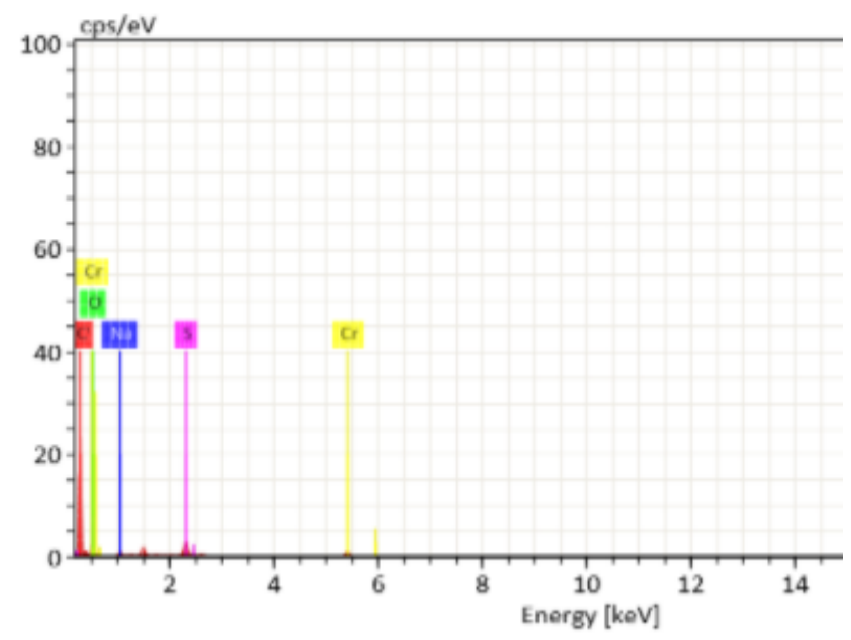

\begin{tabular}{|c|c|c|c|c|c|c|}
\hline Element & At. No. Netto & $\begin{array}{l}\text { Mass } \\
{[\%]}\end{array}$ & $\begin{array}{c}\text { Mass Norm. } \\
{[\%]}\end{array}$ & $\begin{array}{l}\text { Atom } \\
{[\%]}\end{array}$ & $\begin{array}{l}\text { abs. error [\%] } \\
\text { (1 sigma) }\end{array}$ & $\begin{array}{l}\text { rel. error [96] } \\
\text { (1 sigma) }\end{array}$ \\
\hline Carbon & 682579 & 68.19 & 68.19 & 74.78 & 7.79 & 11.43 \\
\hline Oxygen & 821457 & 29.19 & 29.19 & 24.03 & 3.74 & 12.82 \\
\hline Sodium & 115014 & 1.28 & 1.28 & 0.73 & 0.11 & 8.83 \\
\hline Sulfur & 1615656 & 0.72 & 0.72 & 0.30 & 0.05 & 7.18 \\
\hline \multirow[t]{2}{*}{ Chromium } & $24 \quad 6974$ & 0.62 & 0.62 & 0.16 & 0.04 & 7.07 \\
\hline & Sum & 100.00 & 100.00 & 100.00 & & \\
\hline
\end{tabular}


Figure 5. Chemical Compounds at the edges of the laser cut buffalo leather sample

Figure 6 shows the change in level of composition of the compounds. It is evident that the level of carbon has increased while the level of oxygen has reduced due to the thermal effect.

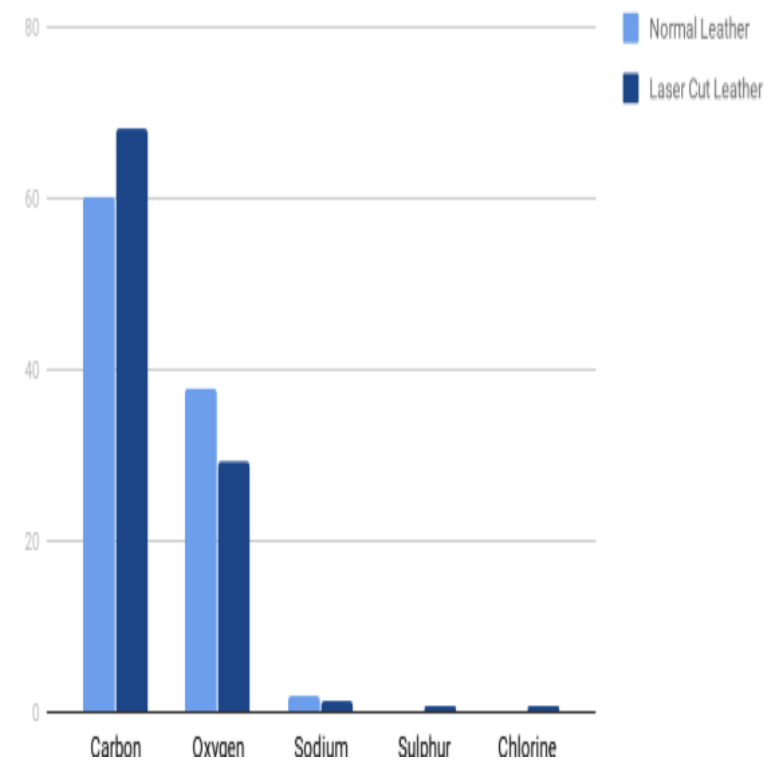

Figure 6. Manual Cut Leather v/s Laser cut Leather

\section{RESULTS AND DISCUSSION}

The images were processed for buffalo leather of two different thickness. From the figures as seen below, figure 1 to figure 4 the thickness of the leather is $1.4 \mathrm{~mm}$ and, figure 5 and figure 6 the thickness is about $1 \mathrm{~mm}$. The images were captured using a USB Digital Microscope and converted into a binary image to calculate the percentage of carbonization on the laser cut edges. To determine this a normal hand cut leather edge was first viewed. The image was captured and converted into binary as seen in Figure 7. Each image captured per frame covers $4 \mathrm{~mm}$ length of the leather. The images captured are magnified nearly 10 times the actual size.

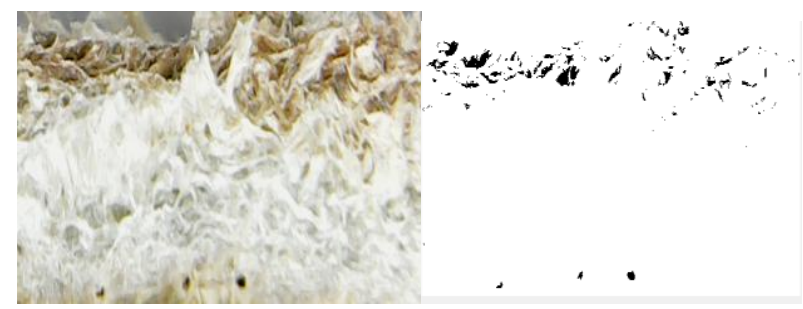

Figure 7. Sample1

Based on the binary image obtained in Figure 7 the binary conversion threshold for that particular leather was set. The edges of other samples cut using the laser was captured and converted. The percentage of carbonization hence was determined based on the binary matrix obtained by conversion of the images. Various other samples and their converted binary images are shown below in figures 8 and 9 .

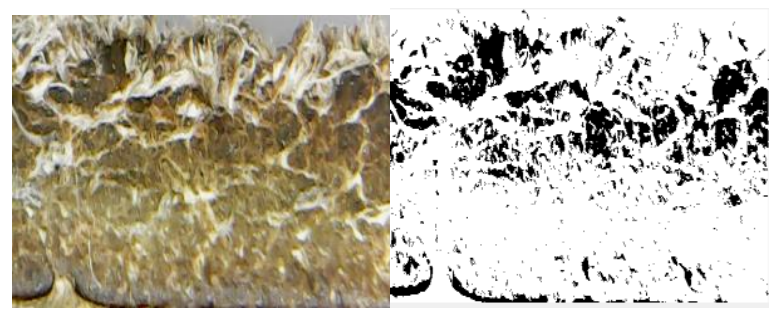

Figure 8. Sample2

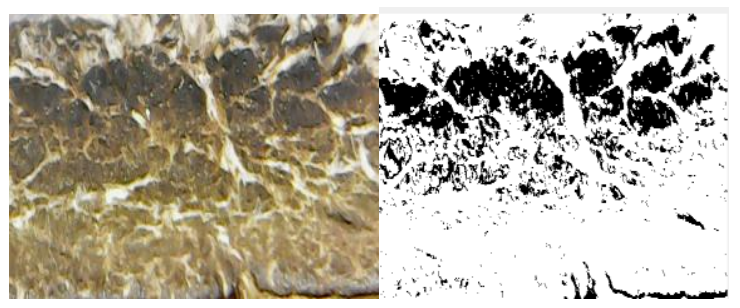

Figure 9. Sample 3

Another leather of different color and thickness $1 \mathrm{~mm}$ was captured similarly. The edge cross section of the uncut leather and the binary image is seen below in Figure 10. A new binary threshold was set for this leather type. The laser cut edges of this leather was captured and processed as seen in Figure 11 to determine the percentage of carbonization.

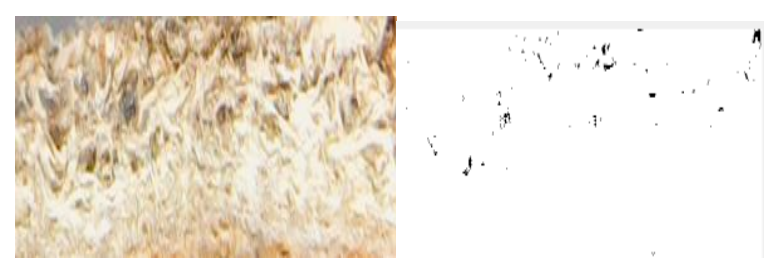

Figure 10. Sample 4

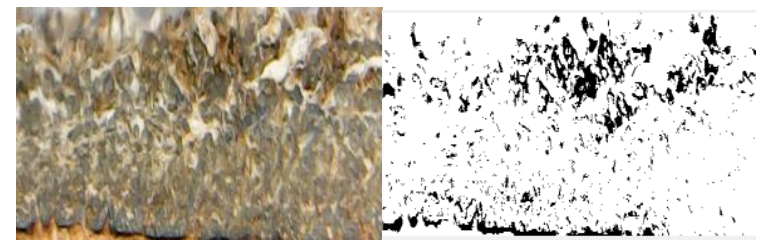

Figure 11. Sample 5

On running the MATLAB code for the images as seen above the percentage of carbonization was determined as shown below in Table 4 . The percentage of heat affected zone corresponds to the percentage of carbonization.

\begin{tabular}{|l|l|l|}
\hline $\begin{array}{l}\text { Sample } \\
\text { No. }\end{array}$ & $\begin{array}{c}\text { \% Of Heat Affected } \\
\text { Zone }\end{array}$ & \% Of Unaffected Zone \\
\hline 1 & 32.6 & 67.4 \\
\hline 2 & 27.4 & 72.6 \\
\hline 3 & 26 & 73.9 \\
\hline 4 & 33 & 66.8 \\
\hline 5 & 29.6 & 70.4 \\
\hline
\end{tabular}

Table 4. Determining the percentage of (HAZ) Heat Affected Zone 


\section{REFERENCES}

1. Alexander Stepanov, Matti Manninen, Inni Pärnänen, Marika Hirvimäki, Antti Salminen " Laser cutting of leather: tool for industry or designers?"15th Nordic Laser Materials Processing Conference, Nolamp 15, 25-27 August

2. K.Hoang, A.Nachimuthu, "Image processing techniques for leather hide ranking in the footwear industry", The University of New South Wales Sydney,2052, Australia.

3. L. Duval1,3, M. Moreaud1, C. Couprie1, D. Jeulin2, H. Talbot3, J. Angulo2, "Image Processing for Materials Characterization, issues, Challenges and opportunities", IEEE International Conference on Image Processing (ICIP) 2014.

4. Mohamed Rafiuddin and Dr.G.Satyanarayana, "Challenges in Exports: A Study of India's Leather Industry”, International Conference on Science Engineering and Management Research 2014.

5. Murali Krishna Kasi,Member,J Bhaskara Rao ,Vijay Kumar Sahu, "Identification of Leather Defects Using an Auto adaptive Edge Detection Image Processing Algorithm", 2014 International Conference on High Performance Computing and Applications (ICHPCA).

6. Parag Kohli and Ms. Shalvi Garg," Leather QualityEstimation Using an Automated Machine Vision System ”,Sangrur, Punjab INDIA Volume 6, Issue 3,May. - June. 2013, PP 44-47.

7. S.Vasanth and T.Muthuramalingam, "A study of Machinability of leather using CO2-Based Laser beam Machining process", Advances in Manufacturing Processes pp 239-244,11 September 2018

8. Umar Farooq, Muhammad Usman Asad, Faiqa Rafiq, Ghulam Abbas, Athar Hanif,"Application of Machine Vision for Performance Enhancement of Footing Machine Used in Leather Industry of Pakistan" Islamabad, Pakistan 10-12 September 2013.

9. U Venkateswarlu1*, M Muthukrishnan1, R Ramesh2 and NK Chandrababu2,"Effect of CO2 Laser on morphological properties of Leather",International Research Journal of Engineering and Technology (IRJET), Volume: 02 Issue: 06,Sep-2015.

10. Nasim, H., Jamil, Y.: Diode lasers: From laboratory to industry. Opt. Laser Technol. 56, 211-222 (2014)

\section{AUTHORS PROFILE}

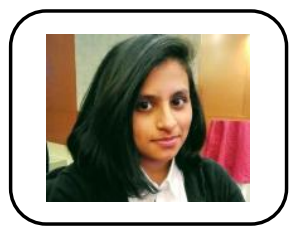

Karen Vanessa Pennefather is an $\mathrm{IV}^{\text {th }}$ year Mechatronics student in SRM Institute of Science and Technology, Kattankulathur .Her region of interest lies in automation and control system.

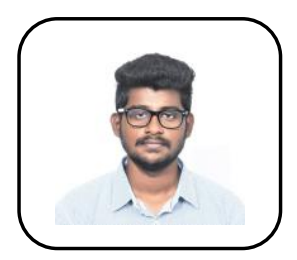

Rajesh Masilamani $\mathbf{A}$ an $\mathrm{IV}^{\text {th }}$ year Mechatronics student in SRM Institute of Science and Technology, Kattankulathur.

Vasanth Swaminathan received the B.E. degree in electronics and communication engineering

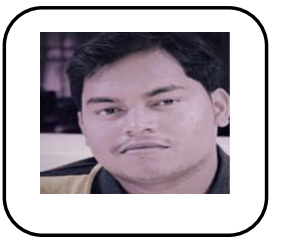
from the Anjalai Ammal Mahalingam Engineering College, Thanjavur, in 2010, and the M.E degree in mechatronics from the Madras Institute of Technology, Anna University, Chennai, in 2012. 